\title{
The Effect of Complex System 's Synergy on the Operational Capability of Weapon Equipment System
}

\author{
Xin-hua HE ${ }^{1, \text { a }}$, Wei-chao ZHANG ${ }^{2, b}$, Qiang Qu ${ }^{3, c}$, Wan-lin LU ${ }^{4, d}$
}

1Department of Information Engineering, Academy of Armored Force Engineering, Beijing, China;

${ }^{2}$ Department of Information Engineering, Academy of Armored Force Engineering, Beijing, China;

${ }^{3}$ Department of Information Engineering, Academy of Armored Force Engineering, Beijing, China;

${ }^{4}$ Troop No.66133 of PLA, Beijing, China

a hxh717495@sina.com, b342722719@qq.com, c535565543@qq.com,d786164744 @qq.com

\section{Key words: Synergy; Combat capability; Weapon system; Neural network model}

Abstract: The synergy of complex systems refers to the coherence between constituent elements, which represents the nature of the coordination and cooperation of the elements in overall development process. By analyzing it by the BP model, the operational capability of the weapon can be assessed more accurately. And through the contrast to consider the synergy and not of the two cases on the impact of combat capability, thus demonstrating the importance of research synergy.

By recognizing and mastering the inherent law of the development of weapons and equipment system, and based on the analysis of the inherent characteristics and structure of the combat capability of the weapon equipment based on the cooperative, the quantitative model of the combat capability evaluation is refined. The BP neural network model is constructed and optimized by genetic algorithm. That is, the generalization ability of GA-BP network model is strong, the global search ability is strong, the adaptability is strong and the self-learning ability is strong, and the combat capability of equipment system is evaluated. Finally, the model is verified by concrete example.

\section{The overview of the combat capability of weapon equipment system}

Weapon Equipment System Combat capability is the ability of the weapon equipment system to perform certain combat missions ${ }^{[1]}$. It is generally believed that the combat capability of the weaponry and equipment system is the mapping of the system function of the weapon equipment system, and also the direct external performance of the weapons and equipment system. The combat capability of weaponry and equipment system is the embodiment of the overall combat capability of weapon equipment system ${ }^{[2]}$.

\section{A Brief Introduction to Combat Capability of Weapon System Based on Collaboration}

\section{a) The Concept of Synergy of Weapon Equipment System}

In the military field, synergy is a significant feature of the weapons and equipment system, composed of weapons and equipment system between the various weapons platform in the process of qualitative change in the system, through coordination and complement each other to achieve the overall combat capability of weapons and equipment system, the ability of this interaction to produce far greater than the functional part of the algebra, and ultimately show that each weapon and equipment platform does not have new features ${ }^{[3]}$. And this new ability with the original sub-system does not have some of the features, these features will be more intuitive performance of the strength of combat capability.

\section{b) The formation of Synergy of Weapon Equipment System}

Weapons and equipment system can get more information on high quality through the equipment between the exchange of information and data sharing, and then make more accurate action 
decisions, the greatest degree of avoid their own situation. Equipment information construction war fog and action friction occurred, improve the degree of coordination between the weapons systems [4].

\section{c) The feature of Synergy of Weapon Equipment System}

Based on the coordination of the equipment system combat capability mainly reflects the integrity, self-organization, relevance and other characteristics ${ }^{[5]}$.

Integrity. The weapon system is a complex system, each of its subsystems through the information to achieve cooperation, it produces greater unity of combat capability than its separate role; Self-organization. The weapons and equipment in the absence of external conditions of intervention, in accordance with certain coordination rules automatically form a stable structure or produce a certain relationship; Relevance. Each component of the relationship between the information in order to achieve the coordination of objectives and adjust each other to coordinate with a weapon or weapons system, the state of a dynamic get changed, this will lead to another get changed ${ }^{[6]}$.

\section{Evaluation and Analysis of Operational Ability of Weapon System Based on Collaboration}

As a result of the hierarchical characteristics of the equipment system, so the provisions of intelligence detection capabilities, command and control capabilities, tactical communication capabilities, electronic combat capability, comprehensive support capabilities and fire strike capability for the first level of combat capability indicators, each level of combat capability indicators Step by step refinement, the specific provisions shown in Figure 1.

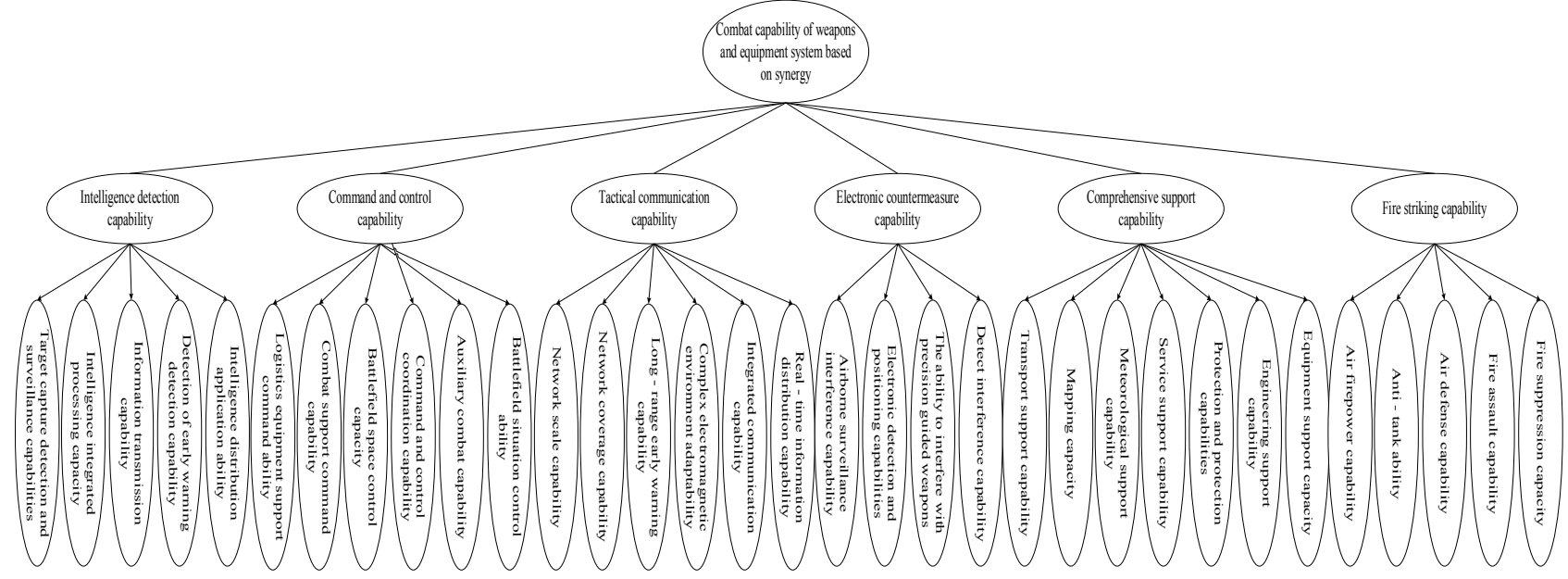

Figure 1 combat capability composition map

A. Intelligence detection capability

The ability to detect intelligence is to monitor, search and track combat environments and operational targets. So our decision-making institutions can control the enemy's control of the enemy's deployment, environmental trends and combat effectiveness and other effective information; effective information dissemination and distribution of the ability to deal with the battlefield environment, early warning and warfare to provide cross-linked guidance Information, and then meet the precision requirements of fire control.

B. Command and control capability

Command and control ability is through the information detection system and tactical communication system to provide information, and other components to establish a suitable system of coordination mechanism, and then effectively organize the implementation of combat operations [7]. Through the real-time evaluation of the battlefield situation can be very good to develop command and coordination mechanism to assist the coordination of the use of battlefield space efficiency and operational support and post-installation command and support institutions of efficient operation. 
C. Tactical communication capability

Tactical communication capability is an important part of the weapon equipment system. It is the carrier of information flow between constituent elements, which provides dynamic, real-time and precise guarantee for combat. Tactical communication capabilities rely on military satellites, wireless base stations and other communications equipment on the battlefield information real-time high-speed distribution of transmission, in the complex electromagnetic environment battlefield, to ensure that the communication system can have a reliable communication capability.

D. Electronic countermeasure capability

The electronic countermeasure is the real-time perception of the electromagnetic situation of the enemy battlefield realized by the electronic countermeasure system. It uses the electronic countermeasure to carry out the attack on the enemy electronic information system and reduce the effectiveness of its battlefield perception, information transmission and weapon guidance capability. E. Comprehensive support capability

Comprehensive support capability is equipment support system for combat to provide a full range of precise comprehensive protection. It mainly includes equipment support ability, engineering support ability, anti-chemical security ability, logistics support ability, meteorological support ability, mapping support ability and transportation support ability.

F. Fire striking capability

Fire strikes are weapons and equipment in order to perform combat missions, it is based on intelligence and battlefield communications real-time data on the enemy's target firepower precision strike ${ }^{[8]}$. One of the main firepower to combat armor-based equipment, the enemy ground forces to implement fire assault, fire suppression.

\section{The Effectiveness Evaluation Model Based on Neural Network}

\section{d) Back Propagation Neural Network}

According to the multi-input and non-linear characteristics of the operational capability evaluation system, BP neural network model is used to abstract the input nodes in the evaluation system into the input points of the neural network. It is a kind of learning feed forward artificial neural network, with the mean square error minimization as the goal to constantly modify the network weights and thresholds, and ultimately to high-precision fitting data. So it is suitable for the study of nonlinear problems such as this article ${ }^{[9]}$. However, because the BP algorithm is slow to learn and there is a possibility of network training failure, it is optimized by genetic algorithm. GA algorithm is an algorithm to realize the global optimal probabilistic search by simulating the natural selection and genetic rule of nature. By optimizing the initial weights and thresholds, the stability of the evaluation results is improved and the accuracy of the evaluation results is improved.

\section{e) Evaluation process}

According to Kolmogorov theorem, the number of hidden layer nodes and the evaluation model are 3-layer neural network model ${ }^{[10]}$. Which specifies that the weight is initialized with a smaller nonzero value $W_{i}(0), W_{i}(t)(1 \leq \mathrm{i} \leq n)$ is the weight of the $\mathrm{i}$-th input at time $\mathrm{t}$, and $\mathrm{C}$ is the threshold at time $t$. Input sample: $\mathrm{X}=\left(\mathrm{X}_{1}, \mathrm{X}_{2}, \ldots, \mathrm{X}_{33}, \mathrm{Y}\right)$ means 33 input samples correspond to the 33 combat capability indicators in the above figure, $\mathrm{Y}$ is the synergistic output: $Y(t)=f\left(\sum_{i=1}^{n} X_{i} W_{i}(t)\right), \quad n=33$. The excitation function is a unipolar S-type function: $f(x)=\frac{1}{1+\mathrm{e}^{-\beta x}}, \beta>0$. Based on the gradient descent BP algorithm, the intensity of the connection of the network is trained, and the mean square error is used as the adaptive function of training ${ }^{[11]}$ : $M S E=\frac{1}{2 M} \sum_{n=1}^{M}\left(u_{n}-\hat{u}_{n}\right)^{2}$. Specify the input of the sample data in the range of input samples of the 
normalized formula for ${ }^{[11]}: y_{(n)}=\frac{x(n)-x_{\min }}{x_{\max }-x_{\min }}$.

The specific steps of the specific algorithm are as follows:

1) connection weights and initialize;

2) given $P$ training samples;

3) use the genetic algorithm to optimize the weight threshold

4) Calculate the output of neurons in each layer;

5) Calculate the output of each neuron;

6) P3 training samples are implemented 4) -5)

7) Calculate the total error and the total correction increment;

8) check whether the total error to meet the accuracy requirements, do not meet the repeat 3 ) - 7).

\section{Example validation}

By comparing the traditional combat capability evaluation model and the evaluation result of the combat capability evaluation model based on the collaborative equipment system, the accuracy of the method of the combat effectiveness evaluation method based on the collaborative equipment system proposed in this paper is judged. The system combat capability value is proportional to the number of evolution The changes are shown in Figure 1. As can be seen from Figure 2, with the increase of the number of system iterations, the evaluation results of the system gradually become stable. Based on the traditional combat capability estimation method and the evaluation result based on the cooperative combat capability evaluation method, the same trend tends to a normal value. The results of the traditional assessment method are 8.479, and the evaluation result based on the collaborative assessment method is 9.112. Through comparison, it can be found that the synergistic effect can reflect the operational capability of the armored brigade more fully.

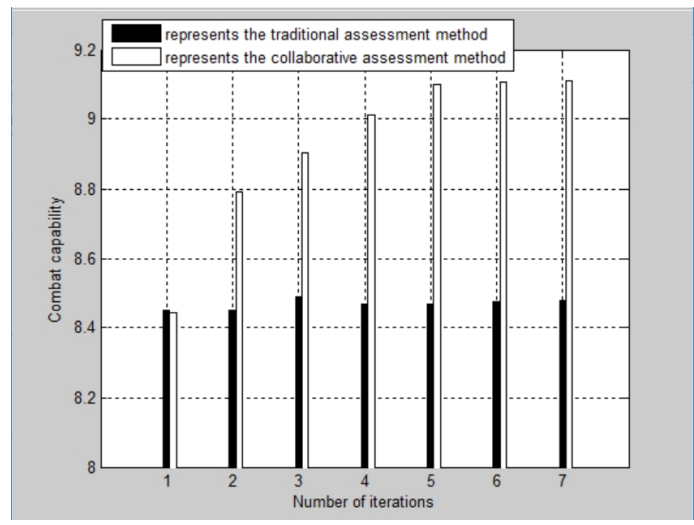

Figure 2 System combat capability and the number of iterations

\section{Conclusion}

Based on the analysis of the structure and function of the weapon equipment system, this paper describes the synergistic mechanism of its combat capability, and analyzes the cooperative relationship between the weapon systems, and simulates it by constructing the GA-BP model.

However, it is a relatively complicated process to analyze the combat capability of the weapon system based on the cooperative. In this paper, there are still some shortcomings in the coordination of the weapon equipment system and the coordination of the coordination index, But also based on the coordination of the operational elements of the role of the relationship between the need to determine a deeper level of research and research.

\section{References}

[1]. Luo Peng cheng, Fu Pan feng, Zhou Jing lun. Weapon equipment system combat capability 
assessment framework [J]. Systems Engineering and Electronics, 2005,: 72-75. [2017-08-30].

[2]. Zhang Di, Guo Qisheng, Li Zhiguo. Based on the ANP weapon equipment system capacity limited level assessment method [J / OL]. System Engineering and Electronics, 2015,37 (04): 817-824. (2014-10-19) [2017-08-30].

[3]. Tao Xiaozhen, Zhao Wendong, Peng Lixian, Tian Chang. A description of service semantic description of battlefield weapon cooperative system based on SOA [J]. Military Communications Technology, 2012,33 (02): 70-76. [2017-08-30].

[4]. Hong Yajun, Chen Yichun, Xu Cheng. Artificial weapon system co - simulation method [J]. Mechanical Design, 2012,29 (01): 87-90. [2017-08-30].

[5]. Zhou Xiaoyu, Liu Sihai. Joint Operations Collaborative Discussion [M]. Beijing: National Defense University Press, 2007.10

[6]. Wang Ziqi, Liu Gaofeng. Evaluation of combat capability of short range anti missile naval weapon system based on SEM [J]. Command Control and Simulation, 2017,39 (04)

[7]. Shu Yu, Tan Yuejin, Li Jufang. Research on the description method of weapon equipment architecture [J]. Systems Engineering and Electronics, 2008, (09): 1704-1707. [2017-08-30].

[8]. Gao Wenkun, Zhang Ke, Yin Zhihong. Research on Agent - based Multi - precision Guided Weapon Cooperative Attack Effectiveness Evaluation [J]. Journal of System Simulation, 2008, (02): 546-549. [2017-08-30].

[9]. Sun Lingfang, Zhou Gaobo, Lin Weijian, Xu Feng. Research on early warning of network public opinion crisis based on BP neural network and genetic algorithm [J]. Journal of Information, 2014,33 (11): 18-24. [2017-08-30]. 